\title{
Radioiodine Scintigraphy with SPECT/CT: An Important Diagnostic Tool for Thyroid Cancer Staging and Risk Stratification
}

\author{
Anca M. Avram \\ Division of Nuclear Medicine/Radiology, University of Michigan Medical Center, Ann Arbor, Michigan
}

\begin{abstract}
CE credit: For CE credit, you can access the test for this article, as well as additional JNMT CE tests, online at https://www.snmmilearningcenter.org. Complete the test online no later than September 2015. Your online test will be scored immediately. You may make 3 attempts to pass the test and must answer $80 \%$ of the questions correctly to receive $1.0 \mathrm{CEH}$ (Continuing Education Hour) credit. SNMMI members will have their CEH credit added to their VOICE transcript automatically; nonmembers will be able to print out a CE certificate upon successfully completing the test. The online test is free to SNMMI members; nonmembers must pay $\$ 15.00$ by credit card when logging onto the website to take the test. Participants who have already taken the exam using JNM and passed cannot retake the exam.
\end{abstract}

Staging and risk stratification predicate the postoperative management of thyroid cancer patients, determining not only the need for ${ }^{131}$ I therapy or alternative options (conservative management without ablation, surgical reintervention, or externalbeam radiation therapy) but also the long-term follow-up strategy. This paper presents the progress made in the field of thyroid cancer imaging by application of SPECT/CT technology to radioiodine scintigraphy in both diagnostic and post-therapy settings and reviews the impact of fusion radioiodine imaging on staging, risk stratification, and clinical management of patients with thyroid cancer. In addition, this paper addresses the role of preablation radioiodine imaging and provides nuclear medicine physicians with the background knowledge required for integrating information from fusion imaging into the clinical and histopathologic risk stratification for developing an individualized treatment plan for patients with thyroid cancer.

Key Words: radioiodine fusion imaging; thyroid cancer; thyroid cancer staging and risk stratification

J Nucl Med Technol 2014; 42:170-180

DOI: 10.2967/jnumed.111.104133

$\mathbf{T}$ he incidence of thyroid cancer continues to rise, with a 2.4-fold increase in incidence since 1975: 44,670 new cases and 1,690 deaths were attributed to thyroid cancer in 2010 (1). Based on histology, thyroid cancers are characterized as $80.2 \%$ papillary thyroid cancers, $11.4 \%$ follicular

\footnotetext{
Received Feb. 9, 2012; revision accepted Mar. 27, 2012.

For correspondence or reprints contact: Anca M. Avram, Division of Nuclear Medicine/Radiology, University of Michigan Medical Center, B1G 505G University Hospital, 1500 E. Medical Center Dr., Ann Arbor, MI 481095028.

E-mail: ancaa@umich.edu

COPYRIGHT (c) 2014 by the Society of Nuclear Medicine and Molecular Imaging, Inc.
}

thyroid cancers, $3.1 \%$ Hürthle cell (or oxyphil) cancers, $3.5 \%$ medullary thyroid cancers, and $1.7 \%$ anaplastic thyroid cancers (2). The 5-y survival rates for well-differentiated thyroid cancer, which includes papillary thyroid cancer, follicular thyroid cancer, and Hürthle cell histologies, are 99.8\% for localized tumors, $97.0 \%$ for tumors with regional metastases, and $57.3 \%$ for tumors with distant metastases (3). In general, stage for stage, the prognosis for papillary thyroid cancer and follicular thyroid cancer is similar; however, certain histologic subtypes of papillary thyroid cancer (such as tall cell variant, columnar cell variant, and diffuse sclerosing variant) and highly invasive follicular thyroid cancer have a worse prognosis (4). Initial therapy for thyroid cancer includes near-total or total thyroidectomy with or without prophylactic or therapeutic central compartment neck dissection to remove the primary tumor and involved cervical lymph nodes (5). Therapeutic lateral neck compartmental dissection is performed for patients with metastatic lateral cervical lymphadenopathy (6-8). Cervical nodal metastases are present in $20 \%-50 \%$ of patients at the time of diagnosis, even with small intrathyroidal tumors (microcarcinomas) (9-12). The completeness of surgical resection is an important determinant of outcome, because residual metastatic lymph nodes represent the most common site of disease persistence or recurrence $(13,14)$.

For many years, postoperative management of thyroid cancer patients included radioiodine administration, followed by a post-therapy scan; however, this approach has been challenged by evidence that remnant ablation does not improve survival in low-risk patients (15-20). The current American Thyroid Association (ATA) Practice Guidelines for Thyroid Cancer Management, published in 2009, emphasize postoperative management according to stage and risk stratification based on clinical-pathologic criteria (i.e., age of patient and the results of surgical pathology) (5). Staging and 


\begin{tabular}{|c|c|}
\hline Score & Characteristics \\
\hline \multicolumn{2}{|c|}{ Primary tumor $(\mathrm{T})$} \\
\hline T1 & $\begin{array}{l}\text { Tumor } \leq 2 \mathrm{~cm} \text {, limited to thyroid; T1a: tumor } \leq 1 \mathrm{~cm} \text { (microcarcinoma); } \\
\text { T1b: tumor }>1 \mathrm{~cm} \text { but } \leq 2 \mathrm{~cm}\end{array}$ \\
\hline T2 & Tumor $>2 \mathrm{~cm}$ but $\leq 4 \mathrm{~cm}$, limited to thyroid \\
\hline T3 & $\begin{array}{l}\text { Tumor }>4 \mathrm{~cm} \text {, limited to thyroid, or any tumor with minimal extrathyroid } \\
\text { extension; for example, extension to sternothyroid muscle or perithyroid } \\
\text { soft tissues }\end{array}$ \\
\hline T4 & $\begin{array}{l}\text { Advanced disease: T4a: tumor of any size extending beyond thyroid capsule } \\
\text { to invade subcutaneous soft tissues, larynx, trachea, esophagus, or recurrent } \\
\text { laryngeal nerve; T4b: tumor invades prevertebral fascia or encases carotid } \\
\text { artery or mediastinal vessels }\end{array}$ \\
\hline \multicolumn{2}{|c|}{ Regional nodes (N) } \\
\hline No & No regional lymph node metastasis \\
\hline N1 & $\begin{array}{l}\text { Regional lymph node metastasis; N1a: metastasis to central neck compartment } \\
\text { (cervical level VI); N1b: metastasis to unilateral, bilateral, or contralateral } \\
\text { lateral neck compartments (cervical levels I, II, III, IV, or V) or retropharyngeal } \\
\text { or superior mediastinal lymph nodes (cervical level VII) }\end{array}$ \\
\hline \multicolumn{2}{|c|}{ Distant metastases (M) } \\
\hline MO & No distant metastasis \\
\hline M1 & Distant metastasis \\
\hline
\end{tabular}

risk stratification are used to assess the prognosis for an individual patient, decide on the use of postoperative ${ }^{131}$ I therapy, decide the frequency and intensity of follow-up, and standardize medical communication. The most commonly used staging system in thyroid cancer is the TNM staging of the American Joint Committee on Cancer/International Union against Cancer, currently in the seventh edition (Tables 1 and 2) (21). Because this staging was developed to predict risk for death-not for recurrence- and does not take into account several independent prognostic variables, the ATA has developed a 3-level risk stratification for patients with thyroid cancer (Table 3) (5).

Staging and risk stratification are used for determining if patients would benefit (and should be referred for) radioiodine therapy: ${ }^{131} \mathrm{I}$ ablation is recommended for patients

TABLE 2

AJCC 7th Edition TNM Staging for Well-Differentiated Thyroid Cancer (21)

\begin{tabular}{|c|c|c|c|}
\hline Stage & $\mathrm{T}$ & $\mathrm{N}$ & $\mathrm{M}$ \\
\hline \multicolumn{4}{|c|}{ Patients $<45$ years old } \\
\hline Stage I & Any $T$ & Any $\mathrm{N}$ & MO \\
\hline Stage II & Any $T$ & Any $\mathrm{N}$ & M1 \\
\hline \multicolumn{4}{|c|}{ Patients $\geq 45$ years old } \\
\hline Stage I & $\mathrm{T} 1$ & NO & MO \\
\hline Stage II & T2 & NO & MO \\
\hline Stage III & $\begin{array}{c}\text { T3 } \\
\text { T1 or T2 or T3 }\end{array}$ & $\begin{array}{l}\text { No } \\
\text { N1a }\end{array}$ & $\begin{array}{l}\text { MO } \\
\text { MO }\end{array}$ \\
\hline Stage IVA & $\begin{array}{c}\mathrm{T} 1 \text { or } \mathrm{T} 2 \text { or } \mathrm{T} 3 \\
\mathrm{~T} 4 \mathrm{a}\end{array}$ & $\begin{array}{l}\mathrm{N} 1 \mathrm{~b} \\
\text { Any N }\end{array}$ & $\begin{array}{l}\text { MO } \\
\text { MO }\end{array}$ \\
\hline Stage IVB & $\mathrm{T} 4 \mathrm{~b}$ & Any $\mathrm{N}$ & MO \\
\hline Stage IVC & Any $T$ & Any $\mathrm{N}$ & M1 \\
\hline
\end{tabular}

with distant metastases, patients with a primary tumor that is grossly invasive or larger than $4 \mathrm{~cm}$, and selected patients with 1to 4-cm tumors confined to the thyroid who have documented node metastases or other high-risk features. The ATA guidelines recommend against ${ }^{131} \mathrm{I}$ ablation in patients with unifocal or multifocal tumors smaller than $1 \mathrm{~cm}$ (microcarcinomas) without high-risk features (5). The most commonly occurring papillary thyroid cancer in the United States is now microcarcinoma: $45 \%$ of tumors in older patients ( $\geq 45 \mathrm{y}$ ) and $34 \%$ of tumors in younger patients $(<45 \mathrm{y})$ are microcarcinomas (22). Although the long-term outcome of papillary microcarcinoma is excellent, it frequently spreads to cervical lymph nodes, as documented in $40.9 \%$ of cases in a series of 445 patients (9), and may occasionally metastasize to distant sites (23-26). The cumulative risk of developing lymph node metastases increases continuously with a primary tumor diameter of $5 \mathrm{~mm}$ or greater; therefore, the concept of "very low risk" papillary thyroid cancer based on tumor size alone becomes questionable (27). When ${ }^{131}$ I therapy is omitted, staging without post-therapy ${ }^{131}$ I whole-body scan (WBS) and stimulated thyroglobulin level has the potential to underestimate the recurrence risk in non-ablated patients. In a slowly growing malignancy such as differentiated thyroid cancer, this risk may become apparent only after long-term follow up (28). This paper addresses a clinically important question: what is the role of radioiodine imaging $\left({ }^{131} \mathrm{I}\right.$ or $\left.{ }^{123} \mathrm{I}\right)$ in risk stratification of patients with thyroid cancer, and what contribution does it bring to completion of staging and to the decision to omit or proceed with ${ }^{131}$ I therapy?

Radioiodine scintigraphy when combined with SPECT/CT has become a powerful diagnostic tool for identification of regional and distant metastases in thyroid cancer. The synergistic combination of functional and anatomic information provided by SPECT/CT has been found to have many advantages 


\begin{tabular}{|c|c|c|c|}
\hline & ATA & Thyroid Cancer Risk Stratification & SNM \\
\hline Very low risk & & $\begin{array}{l}\text { Unifocal or multifocal microcarcinomas }(<1 \mathrm{~cm}) \\
\mathrm{MACIS} \text { score }<6 \text {, or TNM score: } \mathrm{T} 1-2, \mathrm{NO}, \mathrm{MO} \\
\text { In patients }<\mathbf{4 5} \text { years old: tumors }<4 \mathrm{~cm} \text { confined to the thyroid } \\
\text { Excludes tumors with aggressive histology* or vascular invasion }\end{array}$ & $\begin{array}{l}\sqrt{ } \\
\sqrt{ } \\
\sqrt{ } \\
\sqrt{ }\end{array}$ \\
\hline Low-risk (all criteria must be met) & $\begin{array}{l}\sqrt{ } \\
\sqrt{ } \\
\sqrt{ } \\
\sqrt{ } \\
\sqrt{ }\end{array}$ & $\begin{array}{l}\text { In patients }<45 \text { years old: } \mathrm{MACIS}<6 \text {, or TNM score: } \\
\text { any } \mathrm{T} \text { any } \mathrm{N}, \mathrm{MO} \\
\text { In patients } \geq \mathbf{4 5} \text { years old: } \mathrm{MACIS}<6 \text {, or TNM score: T2, NO, M0 } \\
\text { No local or distant metastases } \\
\text { All macroscopic tumor has been resected } \\
\text { There is no tumor invasion of locoregional tissues or structures } \\
\text { The tumor does not have aggressive histology* or vascular invasion } \\
\text { If }{ }^{131} \text { I is given, there is no }{ }^{131} \text { I uptake outside the thyroid bed on } \\
\text { the first post-therapy scan }\end{array}$ & $\begin{array}{l}\sqrt{ } \\
\sqrt{ } \\
\sqrt{ } \\
\sqrt{ } \\
\sqrt{ } \\
\sqrt{ } \\
\sqrt{ }\end{array}$ \\
\hline $\begin{array}{l}\text { Intermediate/Moderate } \\
\text { risk (any criteria) }\end{array}$ & $\begin{array}{l}\sqrt{ } \\
\sqrt{ } \\
\sqrt{ }\end{array}$ & $\begin{array}{l}\text { In patients < } 45 \text { years old: tumors }>4 \mathrm{~cm} \text {; macroscopic }(>1 \mathrm{~cm} \text { ) } \\
\mathrm{N} 1 \mathrm{a} \text { or } \mathrm{N} 1 \mathrm{~b} ; \mathrm{T} 1-3, \mathrm{~N} 1 \mathrm{~b}, \mathrm{M} 0 \\
\text { In patients } \geq 45 \text { years old: } \mathrm{T} 3, \mathrm{~N} 0, \mathrm{M} 0 \text { or } \mathrm{T} 1-3, \mathrm{~N} 1 \mathrm{a}, \mathrm{M0} \\
\mathrm{MACIS} \text { score }>6 \\
\text { Minimally invasive (microscopic capsular, but not vascular } \\
\text { invasion) } \mathrm{FTC}<4 \mathrm{~cm} \\
\text { Tumor with aggressive histology* or vascular invasion } \\
\text { Microscopic invasion of tumor into the perithyroidal soft tissues } \\
\text { at initial surgery } \\
\text { Cervical lymph node metastases, or }{ }^{131} \text { I uptake outside the } \\
\text { thyroid bed on the post-therapy scan }\end{array}$ & $\begin{array}{l}\sqrt{ } \\
\sqrt{ } \\
\sqrt{ } \\
\sqrt{ } \\
\sqrt{ } \\
\sqrt{ }\end{array}$ \\
\hline High-risk (any criteria) & $\begin{array}{l}\sqrt{ } \\
\sqrt{ } \\
\sqrt{ } \\
\sqrt{ }\end{array}$ & $\begin{array}{l}\text { In patients < } 45 \text { years old: } \mathrm{T} 4 \mathrm{a}-4 \mathrm{~b} \text {, any } \mathrm{N}, \mathrm{M} 0 \text {, or any } \\
\mathrm{T} \text {, any } \mathrm{N}, \mathrm{M} 1 \\
\text { In patients } \geq \mathbf{4 5} \text { years old: any } \mathrm{T}, \mathrm{N} 1 \mathrm{a}-1 \mathrm{~b}, \mathrm{M} 0, \mathrm{AJCC} \text { Stages } \\
\mathrm{IVA}, \mathrm{IVB}, \mathrm{IVC} \\
\mathrm{FTC}>4 \mathrm{~cm} \text {, or macroscopic invasive } \mathrm{FTC} \\
\text { Distant metastases } \\
\text { Macroscopic tumor invasion } \\
\text { Incomplete tumor resection } \\
\text { Thyroglobulinemia out of proportion to what is seen on the } \\
\text { post-therapy scan }\end{array}$ & $\begin{array}{l}\sqrt{ } \\
\sqrt{ } \\
\sqrt{ } \\
\sqrt{ } \\
\sqrt{ }\end{array}$ \\
\hline
\end{tabular}

\footnotetext{
*Aggressive histology includes tall cell, insular, columnar, diffuse sclerosing, trabecular solid, poorly differentiated variants of papillary thyroid carcinoma (PTC) and Hürthle cell variant of follicular thyroid carcinoma (FTC); MACIS: Metastases, Age, Completeness of resection, Invasiveness, Size of tumor; staging system developed at Mayo Clinic (16); TNM: Tumor, Nodes, Metastases, staging system developed by AJCC/UICC (21).
}

over traditional planar imaging in different clinical settings. Optimal coregistration of tomographic volumes of data obtained by $\gamma$-cameras with inline CT, with the patient in the same bed position, allows precise anatomic localization of radioactivity foci. Additional benefits include CT-based attenuation correction and morphologic information from unenhanced CT with reduced milliampere-seconds and kilovoltage settings. The advantages of SPECT/CT have been outlined in several excellent reviews on the clinical applications of hybrid imaging (29-33).

\section{ADVANTAGES, DISADVANTAGES, AND LIMITATIONS OF SPECT/CT}

Advantages of SPECT/CT applied either selectively or routinely to planar scintigraphy studies include accurate anatomic localization and characterization of radioiodine foci as benign (such as thyroid remnant tissue or physiologic activity in normal structures) or malignant in cervical nodal or distant metastases. When unusual radioiodine biodistributions are encountered and a physiologic mimic of disease is suspected, SPECT/CT clarifies the interpretation of planar images, thereby avoiding false-positive diagnoses (Fig. 1). Anatomic coregistration of fused SPECT/CT images allows more precise differentiation between malignant and benign radioactivity distributions (Fig. 2). SPECT/CT can solve difficult diagnostic interpretations and reveal metastatic lesions to unexpected sites or tissues. Due to CT-based attenuation correction, SPECT/CT can reveal more foci of pathologic activity as compared to planar studies. The size 


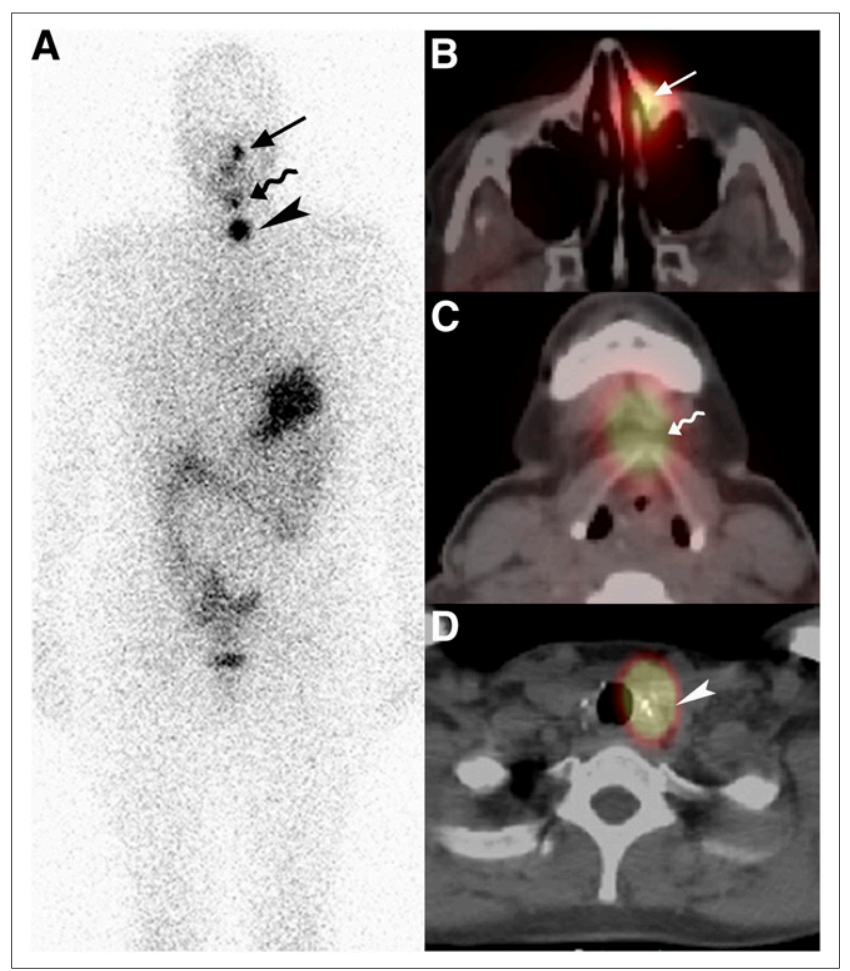

FIGURE 1. Diagnostic ${ }^{131} \mathrm{I}$ scan obtained $24 \mathrm{~h}$ after oral administration of $37 \mathrm{MBq}(1 \mathrm{mCi})$ of ${ }^{131} \mathrm{I}$ in $37-\mathrm{y}$-old woman after total thyroidectomy for multifocal papillary thyroid cancer (two 1.1-cm tumors). Patient had stimulated thyroglobulin level of less than 0.5 $\mathrm{ng} / \mathrm{mL}$; however, measurement was unreliable because specimen contained antithyroglobulin antibodies. (A) Planar whole-body anterior image demonstrates 3 distinct foci of radioactivity, with most superior focus just left of midline in the face (arrow). Two additional foci of activity are seen in neck (wavy arrow and arrowhead). (B) Correlative axial fused SPECT/CT demonstrates localization of superior focus to nasolacrimal duct (arrow), consistent with benign radioiodine accumulation within lacrimal secretions draining into nasal cavity, excluding metastatic osseous lesion to maxilla. (C) Middle focus is centered at tip of hyoid bone and is consistent with thyroglossal duct remnant (wavy arrow), formed by rests of thyroid cells along thyroglossal duct during embryologic migration of thyroid from base of tongue to its final position in lower neck. This is a commonly encountered normal variant, and SPECT/CT improves characterization compared with planar imaging. (D) Lower focus of activity is located paratracheally in left thyroid bed in close association with surgical clips (arrowhead), consistent with thyroid remnant. Focal paratracheal central neck activity can be characterized as benign thyroid remnant when surgical pathology review demonstrates no evidence of tumor extrathyroidal extension and negative surgical excision margins.

and iodine avidity of metastatic lesions can be assessed on the CT and SPECT components, respectively, of SPECT/CT studies, providing information about the likelihood of response to ${ }^{131} \mathrm{I}$ therapy and guiding management decisions on alternative therapeutic options such as surgical excision or external-beam radiation therapy for large or non-iodine-avid metastatic deposits (Fig. 3).

Disadvantages of SPECT/CT include additional imaging time and possible patient discomfort and claustrophobia from lying in a fixed position for approximately $20 \mathrm{~min}$ in

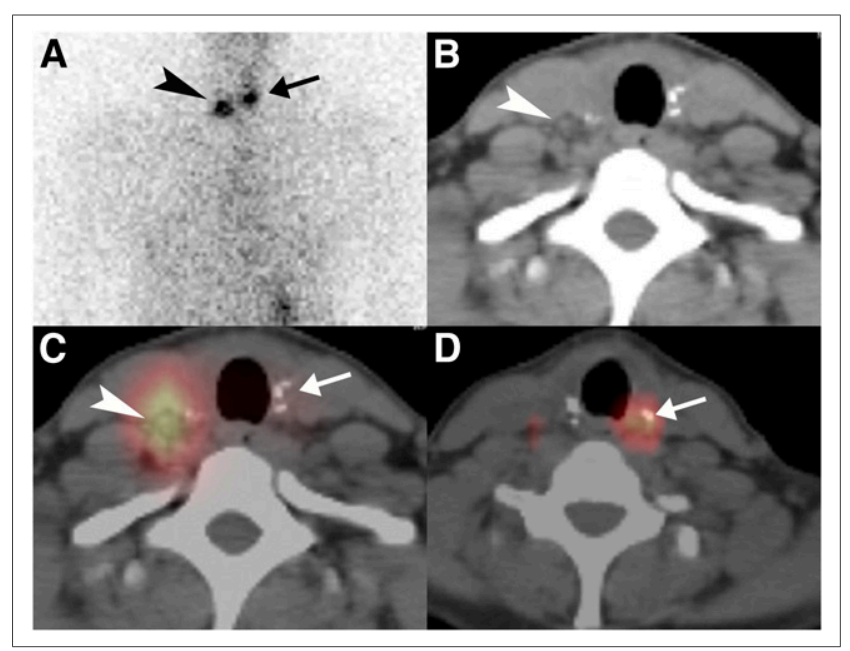

FIGURE 2. Diagnostic ${ }^{131} \mathrm{I}$ scan obtained $24 \mathrm{~h}$ after oral administration of $37 \mathrm{MBq}(1 \mathrm{mCi})$ of ${ }^{131} \mathrm{I}$ in 36 -y-old woman after total thyroidectomy for multifocal papillary thyroid cancer (3.0- and $0.6-\mathrm{cm}$ tumors). Patient's stimulated thyroglobulin level was less than $0.5 \mathrm{ng} / \mathrm{mL}$; however, measurement was unreliable because specimen contained antithyroglobulin antibodies. (A) Planar static anterior view of chest demonstrates 2 foci of activity, one in left neck (arrow) and the other in right lower neck (arrowhead). (B-D) Correlative axial CT (B) and axial fused SPECT/CT (C and D) localize left neck focus to left thyroidectomy bed in close association with surgical clips (arrow), consistent with thyroid remnant. Right neck focus corresponds to $0.5-\mathrm{cm}$ nodule adjacent to right carotid neurovascular bundle (arrowhead), consistent with nodal metastasis.

the tightly enclosed space of the SPECT/CT gantry, and additional radiation exposure from the CT component of the study (1-4 mSv with each acquisition) (30). The benefits and potential risks associated with the study should be assessed for each patient, most importantly in the pediatric population (34).

Recognizing the limitations of SPECT/CT is important: the spatial resolution of SPECT is limited by the partial-volume effect in small lesions; although metastases in normal-sized neck lymph nodes are frequently diagnosed, micrometastatic lesions cannot be detected with SPECT/CT. Similarly, SPECT/ CT is insensitive for the detection of residual locally invasive thyroid cancer after surgery, unless there is gross residual tumor volume or anatomic findings of invasion. Therefore, SPECT/CT radioiodine studies must always be interpreted in the context of the surgical pathology report, which clarifies the presence or absence of tumor invasion into local structures and completion of surgical resection. These elements are of critical importance when one is interpreting the significance of paratracheal central neck activity as benign thyroid remnant versus residual disease. When pathologic review of the surgical resection specimen of total thyroidectomy demonstrates no evidence of tumor extrathyroidal extension and negative surgical excision margins, focal paratracheal central neck activity can be characterized as a benign thyroid remnant. Non-iodine-avid disease, which occurs in approximately $30 \%$ of differentiated thyroid cancer $(35,36)$, may remain undetected on SPECT/CT and lead to 


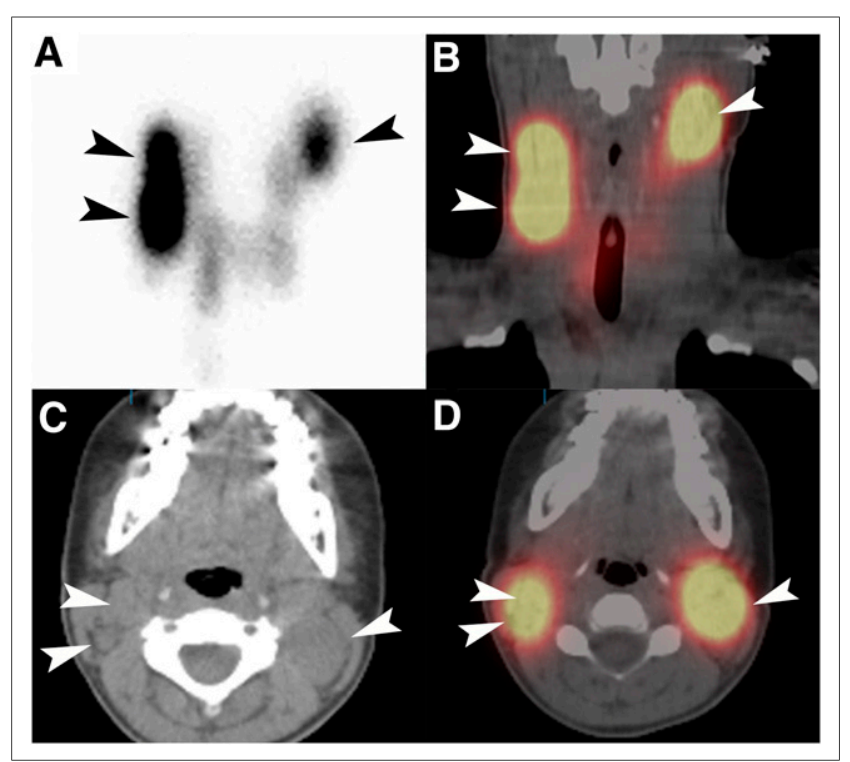

FIGURE 3. Diagnostic ${ }^{131} \mathrm{I}$ scan obtained $24 \mathrm{~h}$ after oral administration of $37 \mathrm{MBq}(1 \mathrm{mCi})$ of ${ }^{131} \mathrm{I}$ in 14 -y-old girl after total thyroidectomy for papillary thyroid cancer extensively involving both lobes and isthmus. Patient's stimulated thyroglobulin level was $406 \mathrm{ng} / \mathrm{mL}$. (A) Pinhole neck image demonstrates longitudinal intense activity in right neck laterally and focal intense activity in left upper neck; in addition, there are several foci of fainter activity in central neck bilaterally. (C-D) Correlative coronal (B) and axial (D) fused SPECT/CT and axial CT (C) demonstrate conglomerate metastatic lymphadenopathy in right cervical levels IIB and III and left cervical level IIB. Due to residual bulky metastases in bilateral neck as demonstrated on planar diagnostic study and measured on SPECT/CT, the patient was referred for reoperative bilateral neck dissection before proceeding with therapeutic ${ }^{131} \mathrm{I}$ administration.

false-negative interpretations. The possibility of noniodine-avid disease needs to be considered in the context of Hürthle cell thyroid cancer, papillary thyroid cancer with unfavorable histology (e.g., tall cell, columnar, or cribriform variants), and poorly differentiated thyroid cancer (such as trabecular, insular, or solid variants). Therefore, the review of histopathology and biochemical data-thyroglobulin, thyrotropin, free thyroxine-remains essential for accurate interpretation of the findings on radioiodine planar and SPECT/CT scintigraphy.

\section{UTILITY OF POST-THERAPY RADIOIODINE SPECT/CT}

The initial reports on the use of SPECT/CT in thyroid cancer described the advantages of SPECT/CT applied to post-therapy ${ }^{131}$ I scintigraphy: in a study evaluating coregistration of separately acquired SPECT and CT data with the aid of external fiducial markers, combined SPECT/CT improved diagnostic evaluation compared with SPECT alone in 15 of 17 patients (88\%) (37). Similarly, in a series of 25 patients with post-therapy ${ }^{131} \mathrm{I}$ scans, SPECT/CT improved diagnostic interpretation compared with planar images in $44 \%$ of radioactivity foci, resulting in a change in management in $25 \%$ of patients (38). In a study of 71 patients, of whom 54 had post-therapy imaging and 17 had diagnostic ${ }^{131}$ I imaging, Tharp et al. reported incremental diagnostic value for SPECT/CT over planar imaging in 57\% of patients. When considering only the group of 17 patients who underwent diagnostic ${ }^{131}$ I studies, the findings on SPECT/CT changed the therapeutic approach for $41 \%$ of these patients (39).

Aide et al. reported that in 55 patients studied with posttherapy ${ }^{131}$ I scans, there were $29 \%$ indeterminate results on planar imaging and only 7\% with SPECT/CT. Of the 16 patients with indeterminate planar scans, reclassification with SPECT/CT as positive or negative for disease correlated with the success or failure of radioiodine treatment at follow-up (40).

One clear advantage of SPECT/CT is its ability to substantially reduce the number of equivocal foci seen on planar imaging alone: Chen et al. reported that SPECT/CT accurately characterized $85 \%$ of foci considered inconclusive on planar imaging, resulting in altered management for $47 \%$ patients (41). Kohlfuerst et al. reported on the impact of post-therapy SPECT/CT in a group of 41 patients: in 33 patients with neck lesions, SPECT/CT changed N status in 12 of 33 patients (36.4\%) and led to a treatment change in 8 of 33 patients (24.2\%); in 19 patients with lesions distant from the neck, SPECT/CT changed $M$ status in 4 of 19 patients (21.1\%) and led to a treatment change in 2 of 19 patients (10.5\%); for the entire group of 41 patients, SPECT/CT led to a treatment change in 10 of 41 patients (24.4\%) (42).

Schmidt et al. reported on nodal staging in 57 patients who underwent planar imaging and SPECT/CT after a first radioablation, and they concluded that SPECT/CT determines lymph nodal status more accurately than planar imaging: 6 of 11 lesions considered nodal metastases on planar imaging were reclassified as benign on SPECT/CT, and 11 of 15 lesions considered indeterminate on planar imaging were reclassified as nodal metastases on SPECT/ CT; SPECT/CT provided information that clarified nodal status in 20 of 57 patients (35\%), which resulted in a change in risk stratification in $25 \%$ of patients (43). Furthermore, the same group determined that the information obtained with ${ }^{131}$ I SPECT/CT performed at the first radioablation can predict the occurrence or persistence of iodine-avid cervical nodal metastases in subsequent follow-up: $94 \%$ of nodal metastatic deposits smaller than $0.9 \mathrm{~mL}$ were eliminated after radioablation, whereas nodal metastases exceeding this size were less likely to completely resolve with ${ }^{131}$ I therapy (44). Consequently, the size information obtained on CT data from the SPECT/CT study can be used for guiding patient selection for further surgery for excision of large metastatic deposits.

SPECT/CT improved anatomic localization of activity foci seen on planar post-therapy ${ }^{131} \mathrm{I}$ imaging in $21 \%$ of cases, as demonstrated by Wang et al. in a study of 94 patients; in addition, SPECT/CT identified new metastatic foci unsuspected on planar imaging in 7\% of patients. Most importantly, the additional information obtained with SPECT/CT resulted in reconsideration of the therapeutic approach in 22 of 94 patients (23\%) (45). 
The use of post-therapy SPECT/CT at the first radioablation has provided information on the incidence of nodal metastases in patients with T1 tumors $(\leq 2 \mathrm{~cm}$, limited to the thyroid). In a large, bicentric study of 151 patients, using a combination of nodal staging based on histopathology (pN1) information (in 46\% patients who underwent surgical neck dissection) and imaging information (SPECT/CT in 54\% patients who did not undergo neck dissection), Mustafa et al. reported that nodal metastases occurred in $26 \%$ of $\mathrm{T} 1$ tumors and in $22 \%$ of microcarcinomas (T1a tumors, $\leq 1.0 \mathrm{~cm}$ ). Regarding nodal staging, SPECT/CT was more accurate than planar imaging in $24.5 \%$ of patients (46).

The use of SPECT/CT technology permitted identification of parapharyngeal metastases in 14 of 561 patients $(2.5 \%)$; in these patients, parapharyngeal metastases were also associated with regional or distant metastases (47).

The effect of post-therapy ${ }^{131} \mathrm{I}$ SPECT/CT on ATA risk classification was assessed by Grewal et al. in a group of 148 intermediate- and high-risk patients (as initially assessed on clinical and histopathology criteria): $74 \%$ of patients underwent a first radioablation, and $26 \%$ of patients received ${ }^{131} \mathrm{I}$ therapy for treatment of recurrent or metastatic disease with rising thyroglobulin levels. SPECT/ CT changed nodal status in $15 \%$ of postsurgical patients and $21 \%$ of patients with suspected disease recurrence or persistence. Based on SPECT/CT findings, ATA risk classification changed in 7 of 109 patients $(6.4 \%)$. Importantly, review of the CT data of the SPECT/CT study identified non-iodine-avid metastases in 32 of 148 patients (22\%) and demonstrated that the size of nodal metastases can be measured on the CT component of SPECT/CT; additional imaging studies were avoided in $48 \%$ of patients (48).

Ciappucinni et al. demonstrated that postablation scintigraphy (planar and SPECT/CT) has prognostic value for predicting the therapeutic outcome of patients with thyroid cancer after total thyroidectomy and first radioablation. The study group comprised 170 patients followed for a median of 29 months (range, 1.5-4.5 y) after initial ${ }^{131} \mathrm{I}$ ablative therapy: 32 patients (19\%) presented with persistent or recurrent disease in subsequent follow-up: 18 patients with nodal metastases, 8 patients with distant metastases, and 6 patients with both nodal and distant metastases. In all patients free of disease at follow-up evaluations, initial postablative SPECT/CT was negative or equivocal for disease. However, SPECT/CT was positive for disease in $78 \%$ of patients identified with persistent or recurrent disease at follow-up; the fact that post-therapy WBS was negative in $22 \%$ patients with persistent or recurrent disease during follow-up points to the presence of non-iodine-avid metastases, also reported by other groups in $20 \%-30 \%$ of patients $(36,48)$. The authors conclude that postablation scintigraphy (planar WBS and SPECT/CT) has 78\% sensitivity and $100 \%$ specificity for predicting recurrent or persistent disease and is the sole independent prognostic variable for disease-free survival (49).
SPECT/CT also improved dual-phase (3 d, $7 \mathrm{~d}$ ) posttherapy ${ }^{131}$ I planar WBS interpretation in a group of 42 patients regarding characterization of focal radioiodine accumulations as benign or malignant (50). Supplemental Table 1 summarizes the results of studies reporting on the use of post-therapy ${ }^{131}$ I scintigraphy with SPECT/CT for accurate lesion localization and characterization (supplemental materials are available online only at http://jnm. snmjournals.org).

\section{UTILITY OF SPECT/CT FOR PROBLEM SOLVING (EVALUATION OF UNUSUAL RADIOACTIVITY DISTRIBUTIONS)}

Unusual patterns of radioiodine biodistribution that could mimic metastatic disease are well recognized and are known to raise a diagnostic dilemma on planar scintigraphy interpretation (51,52). SPECT/CT is an excellent diagnostic tool for rapid evaluation of suspected physiologic mimics and can accurately localize radioiodine distribution to salivary glands, dental fillings, retrosternal goiter, esophageal or airway secretions, hiatal hernias, bowel diverticula, breast, skin contamination, and benign uptake related to radioiodine retention in cysts, bronchiectasis, thymus, benign struma ovarii, or menstruating uterus (53-59). Several reports outline the usefulness of SPECT/CT for solving difficult diagnostic interpretations and revealing unusual metastatic lesions in the liver, kidney, central nervous system, erector spinae muscle, and rectus abdominis muscle (60-64).

\section{UTILITY OF DIAGNOSTIC PREABLATION RADIOIODINE SPECT/CT}

SPECT/CT technology has also been used in conjunction with diagnostic preablation ${ }^{123} \mathrm{I}$ or ${ }^{131} \mathrm{I}$ scans and demonstrated incremental diagnostic information resulting in increased specificity as compared with classic planar scintigraphy. Barwick et al. demonstrated in a group of 79 consecutive patients studied with ${ }^{123}$ I planar imaging, SPECT, and SPECT/CT, that SPECT/CT provided additional diagnostic information in $42 \%$ of patients and provided further characterization in $70 \%$ of foci seen on planar images. The authors calculated the diagnostic performance of SPECT/CT compared with planar scans and SPECT alone: planar studies demonstrated a sensitivity of $41 \%$, specificity of $68 \%$, and accuracy of $61 \%$; SPECT studies demonstrated a sensitivity of $45 \%$, specificity of $89 \%$, and accuracy of 78\%; SPECT/CT provided significant improvement in specificity, with a calculated sensitivity of $50 \%$, specificity of $100 \%$, and accuracy of $87 \%$ (the study group included 11 patients with non-iodine-avid disease demonstrated on anatomic imaging and elevated thyroglobulin levels) (65).

The incremental value of SPECT/CT as compared with planar WBS results from better identification and interpretation of focal uptake, correct anatomic localization and characterization of activity foci, and precise differentiation between metastatic lesions and benign uptake in thyroid 


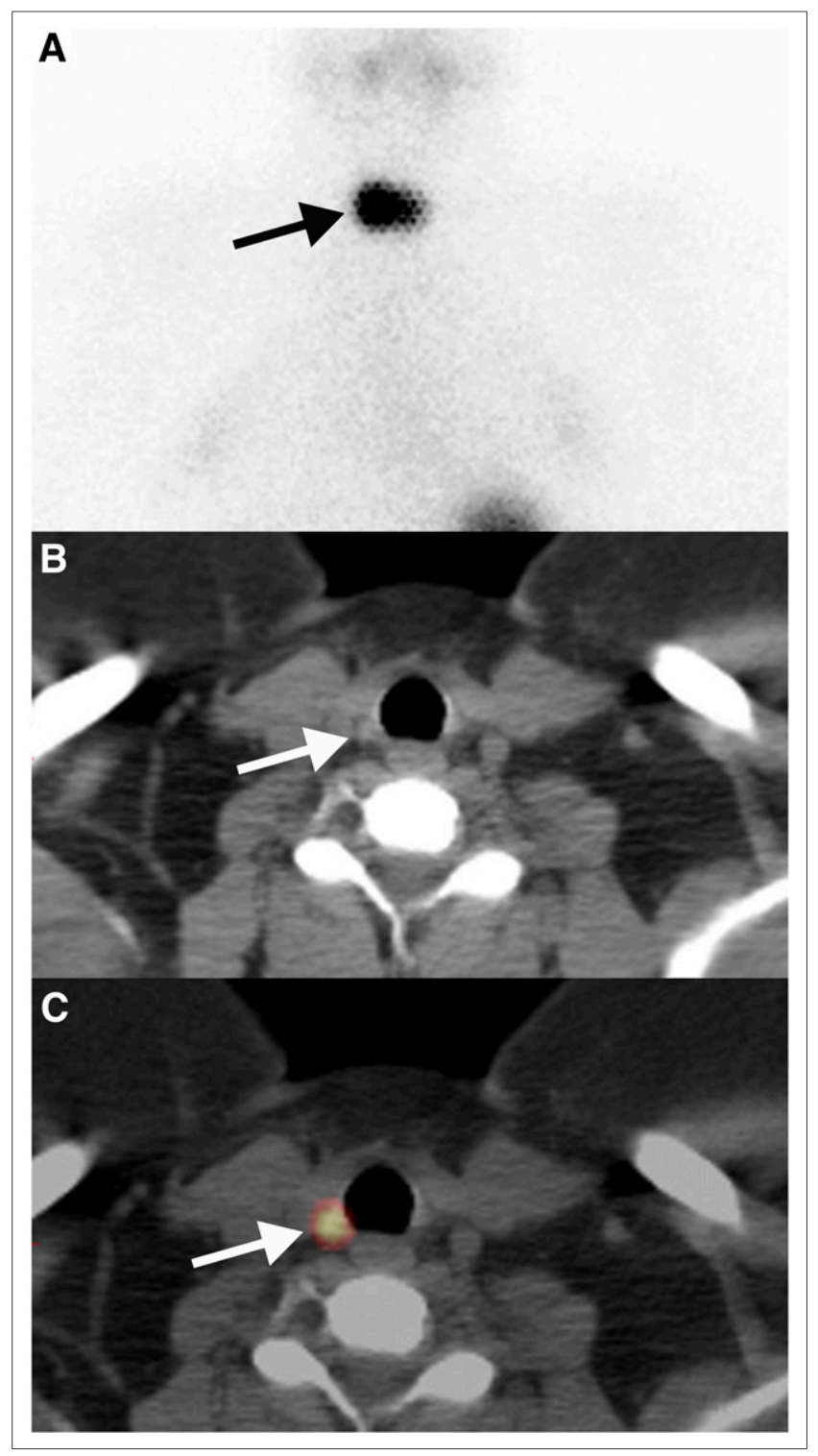

FIGURE 4. Diagnostic ${ }^{131} \mathrm{I}$ scan obtained $24 \mathrm{~h}$ after oral administration of $37 \mathrm{MBq}(1 \mathrm{mCi})$ of ${ }^{131} \mathrm{I}$ in $37-\mathrm{y}$-old woman after total thyroidectomy for goiter with incidental papillary thyroid microcarcinoma (1.3-mm tumor; one metastatic of 3 lymph nodes dissected in the central neck compartment). Patient's stimulated thyroglobulin level was $5.9 \mathrm{ng} / \mathrm{mL}$. (A) Planar static anterior view of chest demonstrates intensely focal central neck activity (arrow). (B and C) Correlative axial CT (B) and axial fused SPECT/CT (C) localizes this activity to $0.4-\mathrm{cm}$ right paratracheal lymph node (arrow) located immediately below cricoid cartilage, consistent with nodal metastasis.

remnant tissue or physiologic radioiodine distribution in normal organs (Fig. 4). In a group of 117 patients studied with planar WBS and SPECT/CT (of whom 108 patients underwent diagnostic ${ }^{123} \mathrm{I}$ and 9 patients underwent posttherapy ${ }^{131}$ I scans), Spanu et al. demonstrated that SPECT/ CT has incremental value over planar scanning in $67.8 \%$ of patients. SPECT/CT identified more foci of pathologic activity (158 foci on SPECT/CT compared with only 116 foci on planar imaging), changed the treatment approach in $35.6 \%$ of patients with disease, and led to avoidance of unnecessary ${ }^{131}$ I therapy in $20 \%$ of patients without disease (66).

Similarly, Wong et al. demonstrated incremental diagnostic value for SPECT/CT over planar imaging for interpretation of $47.6 \%$ of foci seen on WBS in a group of 53 patients studied with a ${ }^{131} \mathrm{I}$ planar and SPECT/CT protocol (the group comprised 47 patients studied with diagnostic preablation scans and 6 patients studied with posttherapy scans). SPECT/CT provided incremental information for interpretation of 53 of 130 neck activity foci (41\%) and 17 of 17 distant foci (100\%) by providing clear anatomic lesion localization and lesion size measurement for predicting the likelihood for response to ${ }^{131}$ I therapy and assessing therapeutic response in subsequent follow-up. Rapid exclusion of physiologic activity or contamination resulted in elimination of equivocal interpretations on planar scans. Reader confidence increased for interpretation of 104 of 147 foci $(71 \%)$ seen on planar images after the review of SPECT/CT (67).

In a group of 48 patients studied with diagnostic preablation ${ }^{131}$ I scans (planar and SPECT/CT protocol) Wong et al. demonstrated that SPECT/CT using low diagnostic activities (37 MBq of ${ }^{131} \mathrm{I}$ ) is feasible and can be used in addition to histopathologic information to complete staging and risk stratification before radioablation (68). The patients were staged according to the TNM system using 3 levels of sequential information: histopathology and chest radiography, planar WBS, and SPECT/CT. The patients were restaged according to the findings on ${ }^{131}$ I scintigraphy (planar, and then planar and SPECT/CT information). Compared with histopathologic analysis, planar imaging and SPECT/CT changed the postsurgical differentiated thyroid cancer stage for $21 \%$ of patients. Identification of unsuspected nodal and distant metastases resulted in prescription of higher therapeutic activities (e.g., $5.5 \mathrm{GBq}$ for regional metastatic disease; 7.4-14.8 GBq and dosimetry calculations for distant metastatic disease), whereas demonstration of only residual functional thyroid tissue in the surgical bed (i.e., thyroid remnant) in the absence of high-risk histopathologic features resulted in prescription of low ${ }^{131}$ I activity (e.g., $1.1 \mathrm{GBq}$ ). Information obtained with diagnostic preablation planar WBS and SPECT/CT scans changed the prescribed radioactivity in 58\% patients, as compared with initially proposed therapy based on histopathologic risk stratification alone (68). In a larger cohort of 320 patients studied with preablation ${ }^{131} \mathrm{I}$ scans, diagnostic preablation ${ }^{131}$ I planar and SPECT/CT scintigraphy detected regional nodal metastases in $22 \%$ and distant metastases in $8 \%$ of patients, leading to upstaging of $4 \%$ of young patients $(<45$ y old) and $25 \%$ of older patients ( $\geq 45$ y old), as compared with pTNM staging based on surgical pathology alone (69).

Supplemental Table 2 summarizes the results of studies reporting on the use of diagnostic preablation ${ }^{123} \mathrm{I} /{ }^{131} \mathrm{I}$ scintigraphy with SPECT/CT for accurate lesion localization and characterization. 
The use of SPECT/CT technology in the setting of diagnostic preablation radioiodine scintigraphy allows recommended staging and risk stratification for patients with thyroid cancer (5) before management decisions. The advantages are the prescription of appropriately higher activities delivered at the first ${ }^{131} \mathrm{I}$ therapy for high-risk patients, when the iodine-concentrating ability of the tumor is presumably highest, and reduction of activity prescribed for radioablation of thyroid remnants. The patients who are considered for management without radioablation due to low-risk features on histopathology may particularly benefit from a postoperative diagnostic scan to exclude the presence of regional or distant metastases and afford the opportunity of obtaining a baseline stimulated thyroglobulin measurement (Fig. 5).

The information obtained with diagnostic radioiodine scans has the potential to impact staging and risk stratification, the decision to proceed with, or to omit ${ }^{131}$ I therapy, and the long-term follow-up strategy. For making a rational decision on the prescribed therapeutic ${ }^{131}$ I activity, the primary goal of ${ }^{131} \mathrm{I}$ therapy should be determined before ${ }^{131} \mathrm{I}$ dose administration and must take into account clinical and histopathologic information and the findings on preablation radioiodine scintigraphy for each individual patient. An understanding of the purpose of therapeutic ${ }^{131} \mathrm{I}$ administration is essential: remnant ablation, defined as the use of ${ }^{131}$ I for elimination of normal residual functional thyroid tissue (thyroid remnant) for facilitating long-term follow-up and to maximize the therapeutic effect of any subsequent ${ }^{131}$ I treatment; adjuvant ${ }^{131}$ I therapy, defined as the use of ${ }^{131}$ I for elimination of suspected but unproven metastatic disease; or targeted ${ }^{131}$ I therapy, defined as the use of ${ }^{131} \mathrm{I}$ for treatment of known local-regional and distant metastases (70). The initial ${ }^{131} \mathrm{I}$ treatment should be targeted at destroying residual or metastatic carcinoma, with the absorbed dose of radiation in the tumor as the best predictor of success for ${ }^{131}$ I therapy.

\section{UTILITY OF RADIOIODINE SPECT/CT FOR LESIONAL DOSIMETRY}

Future directions for the use of hybrid radioiodine imaging involve the use of SPECT/CT to perform lesion-specific dosimetry. Lesion radioiodine uptake and retention can be quantified on SPECT, and tumor volume can be measured on the CT component of the SPECT/CT study, permitting calculation of radiation absorbed dose to tumor (Fig. 6). Follow-up with SPECT/CT can be used to determine therapeutic responses and assess tumor shrinkage. An example of this approach has been reported in a patient with a large skull metastasis causing infringement on the brain (71). Song et al. and Prideaux et al. demonstrated the feasibility of patient-specific 3-dimensional dosimetry using multiple SPECT/CT images, in which the patient's own anatomy and spatial distribution of radioactivity over time are factored into the calculation of radiation absorbed dose to tumor (lesion dosimetry) or to an organ of interest (organ dosimetry). The CT images of SPECT/CT studies are used to provide the density and composition of each voxel for use in a Monte Carlo calculation and to define organs or regions of interest for computing spatially averaged doses. The longitudinal series of SPECT images is used to perform time integration of activity in each voxel and to obtain the cumulated activity per voxel $(72,73)$. The goal of patient-specific voxel-based absorbed dose calculations is better prediction of the biologic effects of radionuclide therapy.

\section{WHEN TO USE SPECT/CT FOR BEST IMPACT IN THYROID CANCER MANAGEMENT}

Performing postoperative diagnostic radioiodine imaging provides the opportunity of identifying patients with unsuspected regional and distant metastases, defines the target of ${ }^{131} \mathrm{I}$ therapy, and is essential for dosimetry calculations if high-dose ${ }^{131}$ I therapy is necessary.

The author of this paper considers that the patients' risk stratification and staging should not be based solely on clinical and histopathologic criteria, but should include specific thyroid cancer imaging to evaluate for the presence of regional and distant metastases. Diagnostic ${ }^{131}$ I scintigraphy (planar studies with or without SPECT/CT) can detect metastases in normal-sized cervical lymph nodes (that would not be visible on postoperative neck ultrasonography), can identify pulmonary micrometastases

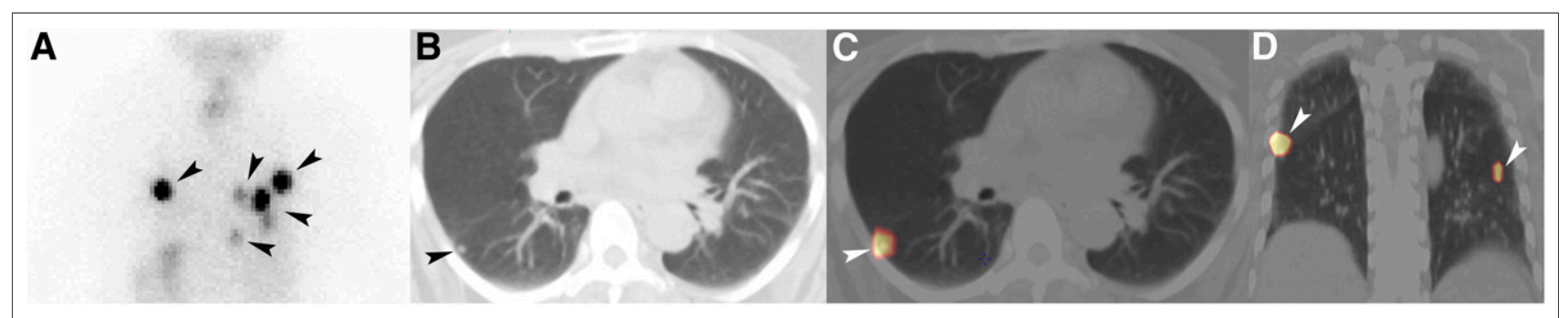

FIGURE 5. Diagnostic ${ }^{131}$ I scan obtained $24 \mathrm{~h}$ after oral administration of $37 \mathrm{MBq}(1 \mathrm{mCi})$ of ${ }^{131} \mathrm{I}$ in 54 -y-old woman after total thyroidectomy for multifocal papillary thyroid microcarcinoma (5 distinct tumors measuring $0.2-0.4 \mathrm{~cm}$ ). Patient's stimulated thyroglobulin level was less than $0.5 \mathrm{ng} / \mathrm{mL}$; however, measurement was unreliable because specimen contained antithyroglobulin antibodies. (A) Planar static posterior view of chest demonstrates multifocal activity within lung fields bilaterally, consistent with pulmonary metastases. (B-D) Correlative axial CT (B) and axial (C) and coronal (D) fused SPECT/CT demonstrate several 0.2- to 0.4-cm iodine-avid pulmonary nodules (arrowheads). 
FIGURE 6. Axial CT (A and $B)$, fused ${ }^{131}$ I SPECT/CT (C), and isodose radiation map (D) in 75-year-old man with solitary calvarial metastasis. Total thyroidectomy with resection of multifocal follicular variant papillary thyroid cancer (1.8- and $0.5-\mathrm{cm}$ tumors) and partial parietooccipital resection occurred 10 y previously; however, the patient presented with recurrent metastasis to skull. Whole-body and lesion dosimetry calculations were performed after oral administration of 37 MBq (1 mCi) of ${ }^{131} \mathrm{I}$. Subsequently, the patient received $7.4 \mathrm{GBq}(200 \mathrm{mCi})$ of ${ }^{131}$ I therapy; stimulated thyroglobulin was $29,124 \mathrm{ng} / \mathrm{mL}$ at time of therapy. (A) Axial CT image demonstrates calvarial metastasis and (B) tumor volumetric measurement of $219 \mathrm{~mL}$ obtained with Vitrea2 software, version 4.1.1.0, Vital Images, Inc. (C) Axial fused SPECT/CT image demonstrates focal radioactivity concentration in tumor. (D) CT image with superimposed isodose levels of cGy in tumor (shaded) and brain. Mean radiation dose to tumor was 1,970 cGy, mainly from beta radiation; gamma (photon) radiation emanating from tumor imparted low level radiation to adjacent brain; mean radiation absorbed dose to brain was 35 cGy. (Reprinted with permission of (71).)

(which are too small to be detected on routine chest radiographs and may remain undetected on CT scans), and can diagnose bone metastases at an early stage before cortical disruption is visible on bone radiographs. Since thyroid cancer osseous metastases are predominantly osteolytic, bone scans with ${ }^{99 \mathrm{~m} T c-m e t h y l e n e}$ diphosphonate or ${ }^{18} \mathrm{~F}$-fluoride may not portray the full extent of skeletal metastatic disease and are most often positive only in larger lesions and advanced-stage disease. More importantly, since ${ }^{131}$ I therapy is most effective for smaller metastatic deposits, early identification of regional and distant metastases is crucial for successful therapy $(44,74)$.

Several studies bring evidence that preablation scans affect the staging of patients with thyroid cancer and that the information obtained with preablation scintigraphy changes management decisions (such as whether to proceed with or to omit therapeutic ${ }^{131} \mathrm{I}$ administration, refer the patient for surgical debulking before ${ }^{131} \mathrm{I}$ therapy, or perform additional imaging studies when non-iodine-avid disease is demonstrated by negative radioiodine findings but elevated thyroglobulin levels). The findings on preablation scans may also alter the prescribed ${ }^{131}$ I activity for thyroid cancer patients, either by adjusting empiric ${ }^{131} \mathrm{I}$ doses or performing dosimetry calculations for maximizing therapeutic ${ }^{131} \mathrm{I}$ activity for treatment of distant metastatic disease. In a study based on a review of 355 diagnostic iodine scans (after administration of 37-148 MBq [1-4 mCi] of either ${ }^{123} \mathrm{I}$ or $\left.{ }^{131} \mathrm{I}\right)$, Van Nostrand et al. demonstrated that in $53 \%$ of cases the findings on preablation scans may alter the patients' management plan (75). Similar conclusions have been reached by other investigators who demonstrated that preablation ${ }^{123}$ I scans provided additional critical information in $25 \%$ of 122 patients, by revealing unsuspected regional or distant metastases indicating the requirement for a significantly higher ${ }^{131}$ I therapy dose, or revealing unexpectedly large thyroid remnants (76). other investigators demonstrated that regional nodal metastases are detected in $22 \%$ of patients, and distant metastases in $8 \%$ of patients (69) and that the information obtained from preablation scans changed the prescribed ${ }^{131}$ I activity in $58 \%$ of patients (68). Diagnostic ${ }^{131}$ I scans are essential when high-dose ${ }^{131} \mathrm{I}$ therapy is considered for treatment of percentage whole-body retentions at $48 \mathrm{~h}$ identify those patients in whom prescribed ${ }^{131}$ I activity may be increased or should be decreased in order not to exceed the maximum tolerated activity $(77,78)$.

\section{CONCLUSION}

The use of radioiodine SPECT/CT has been reported to change clinical management in significant numbers of patients, both when used routinely on all consecutive patients and when used on selected patients with inconclusive planar images. Proposed changes in management include: deciding whether to give or withhold radioiodine treatment, indicating and guiding the extent of surgery, selecting patients for external-beam radiation therapy, and indicating the need for alternative imaging strategies such as ${ }^{18} \mathrm{~F}-\mathrm{FDG}$ PET. Depending on patients' clinical context, the timing of the radioiodine scan (preablation or post-therapy), and the therapeutic protocols at each institution, change in management has been reported in $11 \%(65), 23 \%$ (45), $24 \%$ (42), $25 \%$ (38), $36 \%$ (66), $41 \%$ (39), $47 \%$ (41), and $58 \%(68)$ of patients in various studies.

SPECT/CT is a powerful diagnostic tool that allows accurate anatomic localization and characterization of radioiodine foci and has substantially improved the interpretation of classic radioiodine scintigraphy. SPECT/CT contributes to completion of staging for patients with
Using diagnostic ${ }^{131} \mathrm{I}$ planar imaging and SPECT/CT, metastatic thyroid cancer: dosimetry calculations based on 
thyroid cancer by improved characterization of $\mathrm{N}$ and $\mathrm{M}$ scores. The information obtained with planar imaging and SPECT/CT impacts management in a significant number of patients. The new technology of SPECT/CT has changed the field and should lead to reassessment of current management protocols and guidelines in thyroid cancer.

\section{REFERENCES}

1. Jemal A, Siegel R, Xu J, Ward E. Cancer statistics, 2010. CA Cancer J Clin. 2010;60:277-300.

2. Hundahl SA, Fleming ID, Fremgen AM, Menck HR. A National Cancer Data Base report on 53,856 cases of thyroid carcinoma treated in the U.S., 1985-1995. Cancer. 1998;83:2638-2648.

3. Simard EP, Ward EM, Siegel R, Jemal A. Cancers with increasing incidence trends in the United States: 1999 through 2008. CA Cancer J Clin. January 4, 2012 [Epub ahead of print].

4. Volante M, Landolfi S, Chiusa L, et al. Poorly differentiated carcinomas of the thyroid with trabecular, insular, and solid patterns: a clinicopathologic study of 183 patients. Cancer. 2004;100:950-957.

5. Cooper DS, Doherty GM, Haugen BR, et al. Revised American Thyroid Association management guidelines for patients with thyroid nodules and differentiated thyroid cancer. Thyroid. 2009;19:1167-1214.

6. Gemsenjäger E, Perren A, Seifert B, Schuler G, Schweizer I, Heitz PU. Lymph node surgery in papillary thyroid carcinoma. J Am Coll Surg. 2003; 197:182-190.

7. Kupferman ME, Patterson M, Mandel SJ, LiVolsi V, Weber RS. Patterns of lateral neck metastasis in papillary thyroid carcinoma. Arch Otolaryngol Head Neck Surg. 2004;130:857-860.

8. Goropoulos A, Karamoshos K, Christodoulou A, et al. Value of the cervical compartments in the surgical treatment of papillary thyroid carcinoma. World J Surg. 2004;28:1275-1281.

9. Mercante G, Frasoldati A, Pedroni C, et al. Prognostic factors affecting neck lymph node recurrence and distant metastasis in papillary microcarcinoma of the thyroid: results of a study in 445 patients. Thyroid. 2009;19:707-716.

10. Grebe SK, Hay ID. Thyroid cancer nodal metastases: biologic significance and therapeutic considerations. Surg Oncol Clin N Am. 1996;5:43-63.

11. Scheumann GF, Gimm O, Wegener G, Hundeshagen H, Dralle H. Prognostic significance and surgical management of locoregional lymph node metastases in papillary thyroid cancer. World J Surg. 1994;18:559-56.

12. Chow SM, Law SC, Chan JK, Au SK, Yau S, Lau WH. Papillary microcarcinoma of the thyroid: prognostic significance of lymph node metastasis and multifocality. Cancer. 2003;98:31-40.

13. Shah MD, Hall FT, Eski SJ, Witterick IJ, Walfish PG, Freeman JL. Clinical course of thyroid carcinoma after neck dissection. Laryngoscope. 2003;113: 2102-2107.

14. Wang TS, Dubner S, Sznyter LA, Heller KS. Incidence of metastatic welldifferentiated thyroid cancer in cervical lymph nodes. Arch Otolaryngol Head Neck Surg. 2004;130:110-113.

15. Hay ID. Selective use of radioactive iodine in the postoperative management of patients with papillary and follicular thyroid carcinoma. J Surg Oncol. 2006; 94:692-700.

16. Hay ID, Thompson GB, Grant CS, et al. Papillary thyroid carcinoma managed at the Mayo Clinic during six decades (1940-1999): temporal trends in initial therapy and long-term outcome in 2444 consecutively treated patients. World $J$ Surg. 2002;26:879-885.

17. Jonklaas J, Cooper DS, Ain KB, et al. Radioiodine therapy in patients with stage I differentiated thyroid cancer. Thyroid. 2010;20:1423-1424.

18. Jonklaas J, Sarlis NJ, Litofsky D, et al. Outcomes of patients with differentiated thyroid carcinoma following initial therapy. Thyroid. 2006;16:1229-1242.

19. Podnos YD, Smith DD, Wagman LD, Ellenhorn JD. Survival in patients with papillary thyroid cancer is not affected by the use of radioactive isotope. J Surg Oncol. 2007;96:3-7.

20. Sacks W, Fung CH, Chang JT, Waxman A, Braunstein GD. The effectiveness of radioactive iodine for treatment of low-risk thyroid cancer: a systematic analysis of the peer-reviewed literature from 1966 to April 2008. Thyroid. 2010;20:12351245.

21. Edge SB, Byrd DR, Compton CC, Fritz AG, Greene FL, Trotti A, eds. AJCC Cancer Staging Manual. 7th ed. New York, NY: Springer; 2010.
22. Hughes DT, Haymart MR, Miller BS, Gauger PG, Doherty GM. The most commonly occurring papillary thyroid cancer in the United States is now a microcarcinoma in a patient older than 45 years. Thyroid. 2011;21:231-236.

23. Hay ID, Grant CS, van Heerden JA, Goellner JR, Ebersold JR, Bergstralh EJ. Papillary thyroid microcarcinoma: a study of 535 cases observed in a 50-year period. Surgery. 1992;112:1139-11467.

24. Hay ID, Hutchinson ME, Gonzalez-Losada T, et al. Papillary thyroid microcarcinoma: a study of 900 cases observed in a 60-year period. Surgery. 2008;144:980-987.

25. Strate SM, Lee EL, Childers JH. Occult papillary carcinoma of the thyroid with distant metastases. Cancer. 1984;54:1093-1100.

26. Ross DS, Litofsky D, Ain KB, et al. Recurrence after treatment of micropapillary thyroid cancer. Thyroid. 2009;19:1043-1048.

27. Machens A, Holzhausen HJ, Dralle H. The prognostic value of primary tumor size in papillary and follicular thyroid carcinoma. Cancer. 2005;103:2269-2273.

28. Dietlein M, Verburg FA, Luster M, Reiners C, Pitoia F, Schicha H. One should not just read what one believes: the nearly irresolvable issue of producing truly objective, evidence-based guidelines for the management of differentiated thyroid cancer. Eur J Nucl Med Mol Imaging. 2011;38:793-798.

29. Bockisch A, Freudenberg LS, Schmidt D, Kuwert T. Hybrid imaging by SPECT/ $\mathrm{CT}$ and PET/CT: proven outcomes in cancer imaging. Semin Nucl Med. 2009;39:276-289.

30. Buck AK, Nekolla SG, Ziegler SI, Drzezga A. SPECT/CT. J Nucl Med. May 14, 2009 [Epub ahead of print].

31. Delbeke D, Schoder H, Martin WH, Wahl RL. Hybrid imaging (SPECT/CT and PET/CT): improving therapeutic decisions. Semin Nucl Med. 2009;39: 308-340.

32. Even-Sapir E, Keidar Z, Bar-Shalom R. Hybrid imaging (SPECT/CT and PET/ CT): improving the diagnostic accuracy of functional/metabolic and anatomic imaging. Semin Nucl Med. 2009;39:264-275.

33. Mariani G, Bruselli L, Kuwert T, et al. A review on the clinical uses of SPECT/ CT. Eur J Nucl Med Mol Imaging. 2010;37:1959-1985.

34. Gelfand MJ, Lemen LC. PET/CT and SPECT/CT dosimetry in children: the challenge to the pediatric imager. Semin Nucl Med. 2007;37:391-398.

35. Min JJ, Chung JK, Lee YJ, et al. Relationship between expression of the sodium/ iodide symporter and ${ }^{131} \mathrm{I}$ uptake in recurrent lesions of differentiated thyroid carcinoma. Eur J Nucl Med. 2001;28:639-645.

36. Mian C, Barollo S, Pennelli G, et al. Molecular characteristics in papillary thyroid cancers (PTCs) with no ${ }^{131} \mathrm{I}$ uptake. Clin Endocrinol (Oxf). 2008; 68:108-116.

37. Yamamoto Y, Nishiyama Y, Monden T, Matsumura Y, Satoh K, Ohkawa M. Clinical usefulness of fusion of ${ }^{131} \mathrm{I}$ SPECT and CT images in patients with differentiated thyroid carcinoma. J Nucl Med. 2003;44:1905-1910.

38. Ruf J, Lehmkuhl L, Bertram H, et al. Impact of SPECT and integrated low-dose $\mathrm{CT}$ after radioiodine therapy on the management of patients with thyroid carcinoma. Nucl Med Commun. 2004;25:1177-1182.

39. Tharp K, Israel O, Hausmann J, et al. Impact of ${ }^{131}$ I-SPECT/CT images obtained with an integrated system in the follow-up of patients with thyroid carcinoma. Eur J Nucl Med Mol Imaging. 2004;31:1435-1442.

40. Aide N, Heutte N, Rame JP, et al. Clinical relevance of single-photon emission computed tomography/computed tomography of the neck and thorax in postablation ${ }^{131} \mathrm{I}$ scintigraphy for thyroid cancer. J Clin Endocrinol Metab. 2009;94:2075-2084.

41. Chen L, Luo Q, Shen Y, et al. Incremental value of ${ }^{131} \mathrm{I}$ SPECT/CT in the management of patients with differentiated thyroid carcinoma. J Nucl Med. 2008;49:1952-1957.

42. Kohlfuerst S, Igerc I, Lobnig M, et al. Posttherapeutic ${ }^{131}$ I SPECT-CT offers high diagnostic accuracy when the findings on conventional planar imaging are inconclusive and allows a tailored patient treatment regimen. Eur J Nucl Med Mol Imaging. 2009;36:886-893.

43. Schmidt D, Szikszai A, Linke R, Bautz W, Kuwert T. Impact of ${ }^{131}$ I SPECT/ spiral CT on nodal staging of differentiated thyroid carcinoma at the first radioablation. J Nucl Med. 2009;50:18-23.

44. Schmidt D, Linke R, Uder M, Kuwert T. Five months' follow-up of patients with and without iodine-positive lymph node metastases of thyroid carcinoma as disclosed by ${ }^{131} \mathrm{I}$-SPECT/CT at the first radioablation. Eur J Nucl Med Mol Imaging. 2010;37:699-705.

45. Wang H, Fu HL, Li JN, Zou RJ, Gu ZH, Wu JC. The role of single-photon emission computed tomography/computed tomography for precise localization of metastases in patients with differentiated thyroid cancer. Clin Imaging. 2009;33:49-54.

46. Mustafa M, Kuwert T, Weber K, et al. Regional lymph node involvement in T1 papillary thyroid carcinoma: a bicentric prospective SPECT/CT study. Eur J Nucl Med Mol Imaging. 2010;37:1462-1466. 
47. Qiu ZL, Xu YH, Song HJ, Luo QY. Localization and identification of parapharyngeal metastases from differentiated thyroid carcinoma by ${ }^{131}$ I-SPECT/CT. Head Neck. 2011;33:171-177.

48. Grewal RK, Tuttle RM, Fox J, et al. The effect of post-therapy ${ }^{131}$ I SPECT/CT on risk classification and management of patients with differentiated thyroid cancer. J Nucl Med. 2010;51:1361-1367.

49. Ciappuccini R, Heutte N, Trzepla G, et al. Postablation ${ }^{131}$ I scintigraphy with neck and thorax SPECT-CT and stimulated serum thyroglobulin level predict the outcome of patients with differentiated thyroid cancer. Eur J Endocrinol. 2011;164:961-969.

50. Wakabayashi H, Nakajima K, Fukuoka M, et al. Double-phase ${ }^{131}$ I whole body scan and ${ }^{131}$ I SPECT-CT images in patients with differentiated thyroid cancer: their effectiveness for accurate identification. Ann Nucl Med. 2011;25:609-615.

51. Shapiro B, Rufini V, Jarwan A, et al. Artifacts, anatomical and physiological variants, and unrelated diseases that might cause false-positive whole-body 131-I scans in patients with thyroid cancer. Semin Nucl Med. 2000;30:115-132.

52. Mitchell G, Pratt BE, Vini L, McCready VR, Harmer CL. False positive ${ }^{131} \mathrm{I}$ whole body scans in thyroid cancer. Br J Radiol. 2000;73:627-635.

53. Wong KK, Zarzhevsky N, Cahill JM, Frey KA, Avram AM. Hybrid SPECT-CT and PET-CT imaging of differentiated thyroid carcinoma. $\mathrm{Br} \mathrm{J}$ Radiol. 2009;82:860-876.

54. Wong KK, Avram AM. Post-therapy I-131 thymic uptake demonstrated with SPECT/ CT in a young girl with papillary thyroid carcinoma. Thyroid. 2008;18:919-920.

55. Dümcke CW, Madsen JL. Usefulness of SPECT/CT in the diagnosis of intrathoracic goiter versus metastases from cancer of the breast. Clin Nucl Med. 2007;32:156-159.

56. Macdonald W, Armstrong J. Benign struma ovarii in a patient with invasive papillary thyroid cancer: detection with I-131 SPECT-CT. Clin Nucl Med. 2007;32:380-382.

57. Thust S, Fernando R, Barwick T, Mohan H, Clarke SE. SPECT/CT identification of post-radioactive iodine treatment false-positive uptake in a simple renal cyst. Thyroid. 2009;19:75-76.

58. Rachinsky I, Driedger A. Iodine-131 uptake in a menstruating uterus: value of SPECT/CT in distinguishing benign and metastatic iodine-positive lesions. Thyroid. 2007;17:901-902.

59. Jong I, Taubman K, Schlicht S. Bronchiectasis simulating pulmonary metastases on iodine-131 scintigraphy in well-differentiated thyroid carcinoma. Clin Nucl Med. 2005;30:688-689.

60. Agriantonis DJ, Hall L, Wilson MA. Utility of SPECT/CT as an adjunct to planar whole body I-131 imaging: liver metastasis from papillary thyroid cancer. Clin Nucl Med. 2009;34:247-248.

61. Aide N, Lehembre E, Gervais R, Bardet S. Unusual intratracheal metastasis of differentiated thyroid cancer accurately depicted by SPECT/CT acquisition after radioiodine ablation. Thyroid. 2007;17:1305-1306.

62. Qiu ZL, Luo QY. Erector spinae metastases from differentiated thyroid cancer identified by I-131 SPECT/CT. Clin Nucl Med. 2009;34:137-140.
63. von Falck C, Beer G, Gratz KF, Galanski M. Renal metastases from follicular thyroid cancer on SPECT/CT. Clin Nucl Med. 2007;32:751-752.

64. Zhao LX, Li L, Li FL, Zhao Z. Rectus abdominis muscle metastasis from papillary thyroid cancer identified by I-131 SPECT/CT. Clin Nucl Med. 2010;35:360-361.

65. Barwick T, Murray I, Megadmi H, et al. Single photon emission computed tomography (SPECT)/computed tomography using iodine-123 in patients with differentiated thyroid cancer: additional value over whole body planar imaging and SPECT. Eur J Endocrinol. 2010;162:1131-1139.

66. Spanu A, Solinas ME, Chessa F, Sanna D, Nuvoli S, Madeddu G. ${ }^{131}$ I SPECT/CT in the follow-up of differentiated thyroid carcinoma: incremental value versus planar imaging. J Nucl Med. 2009;50:184-190.

67. Wong KK, Zarzhevsky N, Cahill JM, Frey KA, Avram AM. Incremental value of diagnostic ${ }^{131}$ I SPECT/CT fusion imaging in the evaluation of differentiated thyroid carcinoma. AJR. 2008;191:1785-1794.

68. Wong KK, Sisson JC, Koral KF, Frey KA, Avram AM. Staging of differentiated thyroid carcinoma using diagnostic ${ }^{131}$ I SPECT/CT. AJR. 2010;195:730-736.

69. Avram AM, Doherty GM, Fig LM, Wong K. Diagnostic 131-I fusion SPECT-CT Imaging in postoperative thyroid cancer patients: what is the impact on staging? [abstract]. Thyroid. 2011;21(suppl 1):193.

70. Van Nostrand D. The benefits and risks of I-131 therapy in patients with welldifferentiated thyroid cancer. Thyroid. 2009;19:1381-1391.

71. Sisson JC, Dewaraja YK, Wizauer EJ, Giordano TJ, Avram AM. Thyroid carcinoma metastasis to skull with infringement of brain: treatment with radioiodine. Thyroid. 2009; 19:297-303.

72. Song H, He B, Prideaux A, et al. Lung dosimetry for radioiodine treatment planning in the case of diffuse lung metastases. J Nucl Med. 2006;47:1985-1994.

73. Prideaux AR, Song H, Hobbs RF, et al. Three-dimensional radiobiologic dosimetry: application of radiobiologic modeling to patient-specific 3-dimensional imaging-based internal dosimetry. J Nucl Med. 2007;48:1008-1016.

74. Durante C, Haddy N, Baudin E, et al. Long-term outcome of 444 patients with distant metastases from papillary and follicular thyroid carcinoma: benefits and limits of radioiodine therapy. J Clin Endocrinol Metab. 2006;91:2892-2899.

75. Van Nostrand D, Aiken M, Atkins F, et al. The utility of radioiodine scans prior to iodine 131 ablation in patients with well-differentiated thyroid cancer. Thyroid. 2009;19:849-855.

76. Chen MK, Yasrebi M, Samii J, Staib LH, Doddamane I, Cheng DW. The utility of I-123 pretherapy scan in I-131 radioiodine therapy for thyroid cancer. Thyroid. 2012;22:304-309.

77. Van Nostrand D, Atkins F, Moreau S, et al. Utility of the radioiodine whole-body retention at 48 hours for modifying empiric activity of 131-iodine for the treatment of metastatic well-differentiated thyroid carcinoma. Thyroid. 2009;19: 1093-1098.

78. Sisson JC, Shulkin BL, Lawson S. Increasing efficacy and safety of treatments of patients with well-differentiated thyroid carcinoma by measuring body retentions of ${ }^{131}$ I. J Nucl Med. 2003;44:898-903. 